\title{
The relationship of the size of the footprint of the fibular graft to the surface area of the vertebral endplate in the reconstruction of the anterior column of the spine
}

\author{
Dr RS Rangongo BSc, MBChB(Medunsa), MMed(Orth)(UP) \\ Department of Orthopaedics, 1 Military Hospital, University of Pretoria \\ Prof MV Ngcelwane MBChB(Natal), FCS(SA)Orth, MSc(Orth)(London) \\ Head: Department of Orthopaedics, Steve Biko Academic Hospital, University of Pretoria \\ Dr FE Suleman MBChB, FCRad(D)SA, MMedRad(D)(UL) \\ Clinical Head of Unit: Department of Radiology, Steve Biko Academic Hospital, University of Pretoria
}

\author{
Correspondence: \\ Prof MV Ngcelwane \\ Department of Orthopaedics \\ Steve Biko Academic Hospital \\ University of Pretoria \\ PO Box 667 \\ 0001 Pretoria \\ South Africa \\ Email: mthunzi.ngcelwane@up.ac.za
}

\begin{abstract}
Introduction: The anterior column of the spine is often destroyed by trauma, infection or tumours. It is reconstructed by using an autograft, allograft or synthetic cages. The fibular autograft provides good strength, incorporates quickly and has less risk of disease transmission, which is a big advantage in communities with a high incidence of HIV.

Various authors cite that its major drawback is the size of its footprint because of the possibility of subsidence. We could not, however, find any literature that measures its size.

Aim: To measure the size of the footprint of the fibular graft in relation to the surface area of the vertebral endplate. The clinical relevance is that it may guide the surgeon in deciding how many struts of the fibular graft to use in reconstructing the anterior column, and also quantifies the statement that the fibular strut has a small footprint.

Material and method: CT angiograms are done frequently for peripheral vascular diseases. These angiograms show CT scan images of the lumbar and thoracic vertebrae, and fibulae of the same patient. We retrospectively examined 60 scans done during the years 2012 and 2013. From the CT scans, we measured the surface area of the endplates of the vertebral bodies of T6, 8, 12, L2, and the surface area of the cut surface of the proximal $10 \mathrm{~cm}, 20 \mathrm{~cm}$ and $30 \mathrm{~cm}$ of the fibular graft, all in square millimetres $\left(\mathrm{mm}^{2}\right)$. We then compared the areas of the vertebral measurements to the area of the fibular graft measurements.

Results: The middle third of the fibular graft had the biggest axial surface area. The ratio of the fibular graft surface area to that of the thoracic vertebral endplate is 1:3-6. These ratios suggest that more than one fibular strut graft is required to reconstruct the anterior column in the thoracic spine.

Conclusion: The results show that the fibular graft is better suited for reconstruction in the upper thoracic spine. Below that more than two struts are required.
\end{abstract}

Key words: vertebral body reconstruction, autograft, fibular graft 


\section{Introduction}

The anterior column of the spine is often destroyed by malignancy, infection, trauma and congenital abnormalities. The gold standard for the reconstruction of the anterior column is the use of autologous bone graft. ${ }^{1,2}$ Over the years other materials such as allograft and metallic cages have become more popular.

Allograft is often preferred in the reconstruction of a destroyed anterior column of the spine. The bones often used are the humerus, femur, tibia or a fibula. The grafts are processed under strict conditions to minimise the risk of disease transmission and immuno-incompatibility. ${ }^{3}$ Allografts are acquired through the bone bank and the quantity is therefore only limited if there are financial constraints or delivery problems. Allografts are procured either from living donors or from human cadavers. Their potential morbidity arises mainly from the possible transmission of pathogens, particularly viruses. However, processing of the grafts removes blood and bone marrow in which the viral agents reside. ${ }^{3}$ Mechanical performance of the allograft is weakened by the negative effects of tissue processing, fatigue and post-operative fatigue. ${ }^{4}$ Few guidelines exist regarding donor eligibility as to mechanical integrity of the structural allograft. The principal advantage of the allograft is the avoidance of graft harvest morbidity, and its availability in various shapes and sizes. ${ }^{4}$ Current regulations address disease transmission and tissue contamination so that they are minimised. ${ }^{4}$ Choosing between an allograft and autograft on the basis of economic cost is controversial because studies directly comparing these costs are lacking. ${ }^{2}$ The cost of allografts increases in direct proportion to their processing.

The fibula is readily available and easy to access when harvesting it. It is strong and incorporates well with no risk of disease transmission when used as an autograft. ${ }^{5,6}$ The fibula is superior in axial strength. It provides up to $30 \mathrm{~cm}$ of bone which is easily accessible in the prone, supine and lateral positions. It has a low donor-site morbidity and can easily be harvested simultaneously during spine surgery.

It is used extensively in the cervical spine for multiple level reconstruction. Its use is less popular in the thoracic and lumbar spine, mainly because of the risk of subsidence or fracture of the graft.

The size of the fibular footprint has however, to our knowledge, never been quantified.

Dvorak et al. ${ }^{7}$ mention that the footprint or configuration of the implant-bone interface has not been firmly established in thoracic and lumbar anterior column reconstruction.

We undertook this study with the aim of quantifying the size of the footprint of the fibular graft in relation to the vertebral endplate. This information may be useful in helping the surgeon to decide whether it would be feasible to use a fibular graft in thoracic and lumbar spine.

\section{Material}

The study was approved by the ethics committee of our university. It is a retrospective study of all the patients that had an angiogram of the lower limbs in our department of radiology for peripheral vascular diseases during the period January 2012 to December 2013. Our attraction to these angiographic studies is that they show CT scans of the thoracic spine, lumbar spine and fibular graft, all from the same patient at the same time.

We excluded from the study all patients who had had a leg amputation or who had pathology at the level of the relevant vertebral bodies. We reviewed the $\mathrm{CT}$ angiograms of the first 60 patients who met the requirements for the study. The number 60 was informed by the calculations from the bio-statistician to achieve a minimum power that gives significant statistical results.

\section{Method}

A Siemens Somatom 64 slice CT scan was used to perform the angiograms. The patients were scanned in an axial mode, then coronal and sagittal reconstructions were done. A polygon region of interest was used to measure the circumference which was automatically converted to a surface area expressed in $\mathrm{mm}^{2}$. The measurements were done on the General Electric health Picture Archiving and Communication System (PACS).

On the viewing status of the PACS system the coronal and axial views were cross-referenced to enable measurement of the circumference of T6, T8, T12 and L2 endplates. The cross reference between the two views enables one to identify the anatomical location precisely. The vertebrae $\mathrm{T} 6$ and $\mathrm{T} 8$ were chosen because TB of the spine is common in our setting and often affects the anterior elements of the thoracic spine. ${ }^{8}$ The $\mathrm{T} 12$ and L1 vertebra were chosen because the anterior columns in the thoracolumbar spine in our setting are often destroyed by trauma. ${ }^{7}$

The fibula was measured at 10, 20 and $30 \mathrm{~cm}$ from the tip of its head. A ratio was then established between the $20 \mathrm{~cm}$ cut surface area of the fibula and the vertebral endplates' surface area. The results were used to establish a relationship between the size of the fibular graft footprint and the surface area of the vertebral body endplates.

The $20 \mathrm{~cm}$ level was used to calculate the ratio because it represents the middle third of the fibula which is the part that is utilised as a graft.

The statistical package STATA 13 was used for the data analysis to determine a mathematical relationship between the size of the fibular graft and the surface area of the vertebral bodies.

\section{Results}

The total number of 60 subjects consisted of 38 males $(63 \%)$ and 22 females (37\%). The ages ranged from 21 to 84 years, with a mean of 54.7 years. 
The surface area of the endplates increased from $657.37 \mathrm{~mm}^{2}$ at T6 to $1540.21 \mathrm{~mm}^{2}$ at L2. This is seen in both male and female patients, as shown in Table I.

This is a confirmation of the anatomical increase in size of the vertebrae from the thoracic to the lumbar vertebrae.

The fibular surface area results show that the fibula has the greatest cross-sectional area in the middle segment, with a mean of $138.6 \mathrm{~mm}^{2}$ compared to $118.2 \mathrm{~mm}^{2}$ and $127.1 \mathrm{~mm}^{2}$ in the proximal and distal $20 \mathrm{~cm}$ respectively (Table II). The male patients show bigger surface areas at all three levels examined.

Table III shows the ratio of the surface area of the endplate of the various thoracic lumbar and thoracic vertebrae to that of the fibula at $20 \mathrm{~cm}$ from the tip of the fibula. At T6, the p50 ratio is 4.6. This increases to 12.0 at L2. This means that at T6 we need 4.6 fibular struts to cover the surface area of the vertebral endplate, and 12 at L2. We however do not need to cover the whole endplate surface area with bone graft in reconstruction.

Figure 1 shows a comparison of the ratios between male and females. It shows no significant differences between the two genders in the ratios although the surface areas in males are bigger than those of the females.

\section{Discussion}

Bone grafting procedures are very common in orthopaedics. A retrospective study in Belgium found the procedures to be $13.6 \%$ of all orthopaedic operations in a 3-year period. ${ }^{9}$ Autograft bone graft is considered the gold standard in bone grafting and it is associated with higher rates of union as compared with allograft.

There is currently a trend for surgeons to use allografts as substitutes for autografts due to their quantity, availability and decrease of theatre time. ${ }^{2,3}$ The increasing interest in bone allografts is due to the development of bone banks in many countries and the high safety measures that are maintained in allograft preparation.

The choice of autograft vs allograft depends on surgeon experience, the number of levels being treated, the patient's concern about potential donor site morbidity and other coexisting medical conditions.

The fibular autograft is readily available and easy to access when harvesting. It is strong and incorporates well with no risk of disease transmission. ${ }^{5,6}$ It is used extensively in the cervical spine for multiple level reconstruction. Its use is less popular in the thoracic and lumbar spine.

The middle third of the fibula is typically harvested because of its tubular character and strength. It has the biggest surface area when compared to the proximal and distal parts, as shown in Table II. Anatomically the fibular shaft provides muscle origin or attachment to muscles of the foot. It has four surfaces according to muscular attachment: extensor surface, peroneus surface, flexor surface and the tibialis posterior surface. This explains the strength and increase in size in the middle third segment, as confirmed by this study.
Table I: Analysis of the surface area data of the vertebral endplates and the fibular graft at $20 \mathrm{~cm}$ from the tip of the fibular head, in $\mathrm{mm}^{2}$

\begin{tabular}{|l|r|r|r|r|r|r|r|}
\hline Gender & \multicolumn{2}{c}{ N } & \multicolumn{2}{c}{ Stats } & \multicolumn{2}{c}{ T6 } & \multicolumn{2}{c}{ T8 } & \multicolumn{2}{c}{ T12 } & \multicolumn{2}{c}{ L2 } & Fib 20 cm \\
\hline \multirow{2}{*}{ Female } & \multirow{2}{*}{22} & Mean & 564.414 & 648.982 & 1055.514 & 1262.568 & 123.318 \\
& & SD & 110.553 & 158.864 & 212.732 & 387.065 & 31.331 \\
\hline \multirow{2}{*}{ Male } & \multirow{2}{*}{38} & Mean & 711.197 & 853.524 & 1364.605 & 1792.958 & 147.516 \\
& & SD & 160.639 & 210.853 & 254.717 & 374.301 & 36.864 \\
\hline \multirow{2}{*}{ Total } & \multirow{2}{*}{60} & Mean & 657.377 & 781.692 & 1251.272 & 1540.215 & 138.643 \\
& & SD & 160.066 & 217.332 & 281.705 & 431.939 & 36.604 \\
\hline
\end{tabular}

Table II: Analysis of the combined surface area data of the fibula at different heights

\begin{tabular}{|c|c|c|c|c|c|}
\hline Gender & $\mathbf{N}$ & Stats & Fibula $10 \mathrm{~cm}$ & Fibula $20 \mathrm{~cm}$ & Fibula $30 \mathrm{~cm}$ \\
\hline \multirow{2}{*}{ Female } & \multirow{2}{*}{22} & Mean & 112.818 & 123.318 & 125.068 \\
\hline & & SD & 28.987 & 31.331 & 36.701 \\
\hline \multirow{2}{*}{ Male } & \multirow{2}{*}{38} & Mean & 121.432 & 147.516 & 128.337 \\
\hline & & SD & 33.311 & 36.864 & 26.978 \\
\hline \multirow{2}{*}{ Total } & \multirow{2}{*}{60} & Mean & 118.273 & 138.643 & 127.138 \\
\hline & & SD & 31.819 & 36.604 & 30.633 \\
\hline
\end{tabular}

Table III: Analysis of the combined computed ratio of surface areas in Table I

\begin{tabular}{|c|c|c|c|c|c|}
\hline Gender & Stats & Ratio T6/20 & Ratio T8/20 & Ratio T12/20 & Ratio L2/20 \\
\hline \multirow{6}{*}{ Female } & $\mathrm{N}$ & 22 & 22 & 22 & 22 \\
\hline & Mean & 4.807971 & 5.503932 & 9.064269 & 10.81967 \\
\hline & SD & 1.323247 & 1.602212 & 2.955706 & 4.188323 \\
\hline & Min & 2.881635 & 3.033557 & 5.217913 & 1.022543 \\
\hline & Max & 7.529769 & 8.620899 & 17.45975 & 18.5079 \\
\hline & p50 & 4.654064 & 5.125564 & 8.621651 & 11.56925 \\
\hline \multirow{6}{*}{ Male } & $\mathrm{N}$ & 38 & 38 & 38 & 38 \\
\hline & Mean & 5.105856 & 6.084071 & 9.717685 & 11.97368 \\
\hline & SD & 1.7301 & 1.869948 & 2.689688 & 3.03704 \\
\hline & Min & 2.567442 & 3.512565 & 5.47611 & 7.537051 \\
\hline & Max & 9.783914 & 10.43128 & 16.66825 & 17.71636 \\
\hline & p50 & 4.626639 & 5.928061 & 9.297252 & 12.07476 \\
\hline \multirow{6}{*}{ Total } & $\mathrm{N}$ & 60 & 60 & 60 & 60 \\
\hline & Mean & 4.996632 & 5.871354 & 9.478099 & 11.55054 \\
\hline & SD & 1.587862 & 1.784947 & 2.783373 & 3.513201 \\
\hline & Min & 2.567442 & 3.033557 & 5.217913 & 1.022543 \\
\hline & Max & 9.783914 & 10.43128 & 17.45975 & 18.5079 \\
\hline & p50 & 4.634661 & 5.584677 & 9.247819 & 12.07476 \\
\hline
\end{tabular}




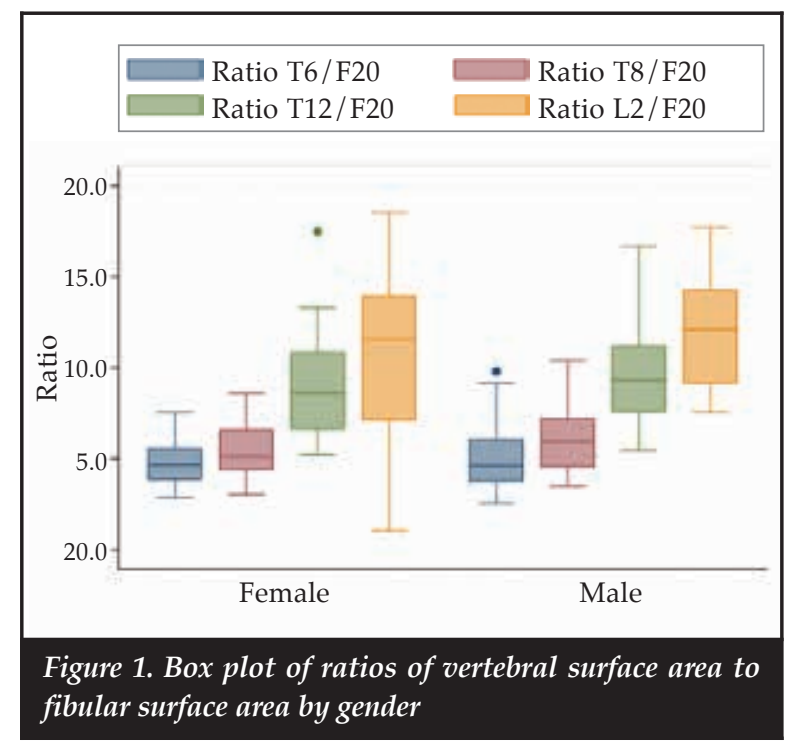

The fibular length is $352.3 \mathrm{~mm} \pm 15.1 \mathrm{~mm} \cdot{ }^{10}$ Its main disadvantage is the small footprint which has been documented. ${ }^{7,11}$ Dvorak et al. ${ }^{7}$ mention that the footprint or configuration of the implant-bone interface has not been firmly established in thoracic and lumbar anterior column reconstruction. Iliac crest and fibular grafts maintain a relatively small area of contact with the endplate and thus may promote penetration through the endplate and subsidence into the vertebral body, resulting in the loss of sagittal alignment. Winters et al. ${ }^{11}$ mention the use of multiple barrel strut grafts of free vascularised fibular graft to reconstruct the thoraco-lumbar anterior column.

In the thoracic and lumbar spine, its use has been less popular because of the size of the footprint. In fact when it is used in the lumbar or thoracic spine, it has been used as two or three struts bundled together. This is because of the size of its footprint. To our knowledge this size has never been evaluated.

Table I in this study shows that the surface area of the endplate of the vertebra increases as one moves from upper thoracic spine to lumbar spine. This is found in both males and females. While one fibular strut is adequate for replacement of the vertebral body in the cervical spine, this study shows why multiple struts are used in the thoracic and lumbar spine.

The relationship between the graft cross-sectional area and the area of the adjacent endplates has so far received no attention in the literature. ${ }^{12}$ Using animals whose spines are comparable to humans, Kubosch et al. ${ }^{12}$ demonstrated that a graft fractured if the graft cross-sectional area was smaller than $21 \%$ of the surface area of the endplates to be fused. The ideal footprint of the implant-bone interface has also not been firmly established. This is evidenced by the wide variability in design among the numerous prosthetic intervertebral devices currently available. ${ }^{7}$
The surface area of a commonly used commercial cage (SynMesh, Synthes ${ }^{\circledR}$ ) for the thoracic vertebrae is $17 \times 22 \mathrm{~mm}$ which is $374 \mathrm{~mm}^{2}$. This is found to be twice the surface area of a fibula at $20 \mathrm{~cm}$ from the tip as demonstrated in Table II (mean $138.64 \mathrm{~mm}^{2}$, SD 36.6). The vertebral endplate surface areas measured a mean of $657.3 \mathrm{~mm}^{2}$ at $\mathrm{T} 6$ and $781.6 \mathrm{~mm}^{2}$ at $\mathrm{T} 8$. We therefore extrapolated that two fibular struts can be used for support in the upper thoracic spine, up to $\mathrm{T} 8$ anterior column reconstruction.

The surface areas at T12 and L2 are so big that it would be impractical to use a fibular graft with a small footprint as evidenced by the calculated ratio to the fibula at $20 \mathrm{~cm}$, as shown in Table III.

It would be technically difficult to use more than two strut grafts and stabilise them on the endplate. The possibility of graft displacement posteriorly and injury to the spinal cord will be increased.

The shortcoming of this study is the limit in application with multilevel anterior column reconstruction. This study does not determine the height of the strut graft needed for a single vertebra and the intervertebral disc. We are therefore not able to determine how many levels of the vertebrae can be reconstructed at the upper thoracic spine. With the fibular graft harvesting and its associated morbidities, it will be impossible to harvest bilaterally to provide enough strut grafts to achieve multilevel reconstruction of the anterior column. The fibula has a dual biomechanical role of providing a site of origin for the muscles and of serving as a rigid body in load transfer. ${ }^{13-15}$ Bilateral harvesting would therefore weaken muscles of the foot.

The study does not aim to replace the present use of allograft as the preferred method of reconstruction. It aims to avail an option for the surgeon who cannot use an allograft for any reason. We used a novel way of determining the footprint of the fibular graft and measuring the vertebral endplate surface area which is non-invasive and reproducible.

\section{Conclusion}

We have been able to evaluate the size of the footprint of the fibular graft and measure the surface area of the thoracic and lumbar vertebral endplate using a novel method, which is the use of a CT angiogram.

Data analysis shows that the fibular graft is big enough to be useful in the upper thoracic spine as two strut grafts. The lower thoracic spine and the lumbar spine require too many struts to be considered practical.

\section{Acknowledgements}

Mr N Malebo, radiographer, for assisting with the measurement of the graft surface area.

Dr S Olorungo, bio-statistician, for helping with the statistical analysis. 


\section{Compliance with Ethics Guidelines}

RS Rangongo, MV Ngcelwane and FE Suleman declare that they have no conflict of interest. This research was approved by the Health Sciences ethics committee of the University of Pretoria, ref. no. 39/2015.

\section{References}

1. Brazenor GA. Comparison of Multisegment anterior cervical fixation using bone strut graft vs a titanium rod and buttress prosthesis. Spine 2007;32(1):63-71.

2. Malloy KM, Hilibrand AS. Autograft versus Allograft in degenerative cervical disease. CORR 2002;394:27-38.

3. Albert A, Leemruse T, Druez V, Delloye C, Cornu O. Are bone autografts still necessary in 2006? A three year retrospective study of bone grafting. Acta Orthop Belg 2006;72:734-40.

4. Kawaguchi S, Hart RA. The need for structural allograft biomechanical guidelines. Journal of the AAOS February 2015; Vol 23(2):119-25.

5. Goldberg VM, Stevenson S. Natural history of autografts and allografts. Clin orthop 1987;225:7-16

6. Whitecloud TS, LaRocca H. Fibular strut graft in reconstructive surgery of the cervical spine. Spine 1976;1:33-43.

7. Dvorak MF, Kwon BK, Fisher CG, Eiserloh HL, Boyd M, Wing PC. Effectiveness of titanium mesh cylindrical cages in anterior column reconstruction after thoracic and lumbar vertebral body resection. Spine 2003;28(9):902-908.

8. Govender S. The outcome of allografts and anterior instrumentation in spinal tuberculosis. CORR May 2002;398:60-66
9. Govender S, Parbhoo AH. Support of the anterior column with allografts in tuberculosis of the spine. JBJS;81-B: 106-109.

10. Nassr A, Khan MH, Ali MH, Espiritu MT, Hanks SE, Lee JY, et al. Donor-site complications of autogenous nonvascularized fibula strut graft. The Spine Journal 2009;9:893-98.

11. Winters HAH, Van Engeland AE, Jiya TU, Van Royen BJ. The use of free vascularised bone grafts in spinal reconstruction. J of Plastic, Reconstructive \& Aesthetic Surgery. 2010;63:516-23.

12. Kubosch D, Milz S, Sprecher CM, Sudkamp NP et al. Effect of graft size on graft fracture rate after anterior lumbar spinal fusion in a sheep model. Injury, int. J. Care Injured 2010;41:768-71.

13. Vail TP, Urbaniak JR. Donor site morbidity with use of vascularized autogenous fibular grafts. JBJS Feb 1996; 78-A (2):204-11.

14. Hughes SS, Pringle T, Phillips F, Emery S. Settling of fibula strut grafts following multilevel anterior cervical corpectomy, a radiographic evaluation. Spine 2006;31:1911-15.

15. Ackerman DB, Rose PS, Moran SL, Dekutoski MB, Bishop AT, Shin AY. The results of vascularised free fibular grafts in complex spinal reconstruction. J Spinal Disord 2011;24(1):70-176.

This article is also available online on the SAOA website (www.saoa.org.za) and the SciELO website (www.scielo.org.za). Follow the directions on the Contents page of this journal to access it. 